\title{
A Practical Method to Determine True Pitching Moment for Internal Balance Wind Tunnel Testing with Longitudinal Variation in Flow Angularity
}

\author{
Kevin W. Kelly ${ }^{1}$ \\ Wichita State University, Wichita, KS, 67260-0093
}

\begin{abstract}
Data reduction and correction checks for Walter $\mathrm{H}$. Beech Wind Tunnel internal balance tests demonstrate an offset in observed pitching moment for model upright and inverted test runs. Root-cause analysis determines longitudinal variation in flow angularity is the primary cause, which is quantified via direct probe measurements. A horizontal tail effectiveness correction is developed to apply the flow angularity variation to a model's longitudinal lifting surfaces, with good resulting upright-to-inverted pitching moment correlation.
\end{abstract}

\section{Nomenclature}

AoA $\quad=$ angle of attack

$C_{L} \quad=$ lift coefficient

$C_{m} \quad=$ pitching moment coefficient

$C_{m, \text { true }}=$ true pitching moment coefficient corrected for observed $h$-tail upflow

$C_{m, i H}=$ change in pitching moment coefficient with change in $h$-tail incidence

$C_{m, \text { ind }}=$ indicated pitching moment coefficient

$F B=W B W T$ "Fighter-Bomber" wind tunnel test model

h-tail = horizontal tail

$M \quad=$ Mach number

NIAR = National Institute for Aviation Research

SATA = Subsonic Aerodynamic Testing Association

WBWT = Walter H. Beech Wind Tunnel

WSU = Wichita State University

$x=$ longitudinal distance in wind axis coordinates (inches; positive forward)

$\alpha_{c} \quad=$ AoA corrected for upflow and wind tunnel effects

$\alpha_{u p} \quad=$ upflow angle

$\Delta C_{m} \quad=$ difference in observed pitching moment for wind tunnel runs of upright and inverted model

$\Delta i_{H} \quad=$ differential $h$-tail upflow angle relative to wing upflow angle

\section{Introduction}

common wind tunnel data corrections check method is the comparison of reduced data for back-to-back upright $\mathbf{A}_{\text {and inverted wind tunnel model runs, when practical. This method is quite easily employed on internal balance }}$ mounting systems with rollheads, and follows a portion of the full tare and interference run set outlined in the classical low-speed wind tunnel corrections of Barlow, et al. ${ }^{1}$. In principle, the full set of wind tunnel flow and boundary corrections - flow angularity, streamline curvature, balance aerodynamic misalignment, etc. - are verified simultaneously by comparison of corrected data plots.

The lift polar is used primarily, where, for a full 3-D model aircraft test, the linear lift region will typically exhibit a direct translation in angle-of-attack (AoA) for equivalent lift coefficient in upright and inverted runs, as in Fig. 1a. Determining half the translation in AoA yields the average upflow angle $\left(\alpha_{u p}\right)$ encountered by the entire model throughout its linear lift region. Direct addition of $\alpha_{u p}$ to all other wind tunnel AoA corrections yields the model's true AoA, $\alpha_{c}$ (similarly, $\alpha_{u p}$ is subtracted from inverted run AoA data). Plots of lift coefficient versus true AoA typically yield very good agreement for an upright and inverted model, as shown in Fig. 1b.

\footnotetext{
${ }^{1}$ Research Engineer, WSU NIAR, 1845 Fairmount, Wichita, KS 67260-0093, AIAA Member
}

American Institute of Aeronautics and Astronautics 


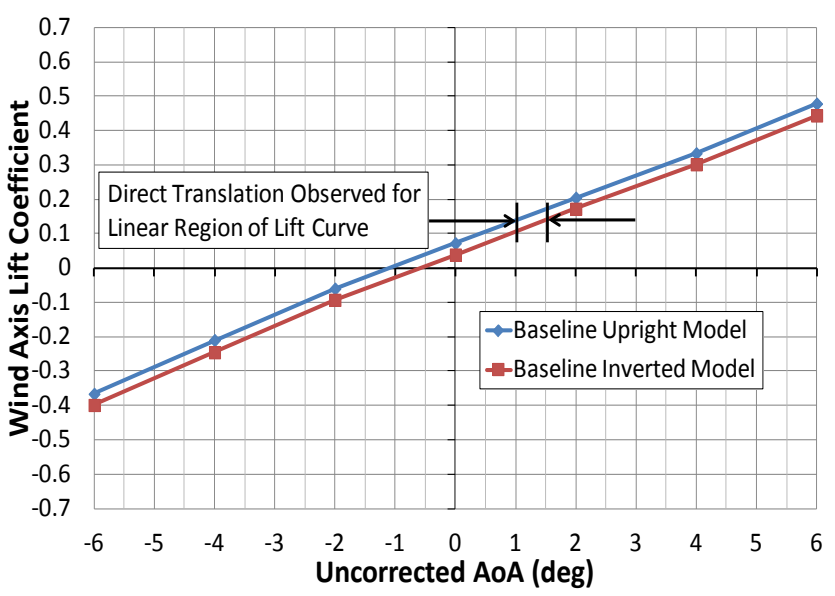

Figure 1a. Typical uncorrected lift curves.

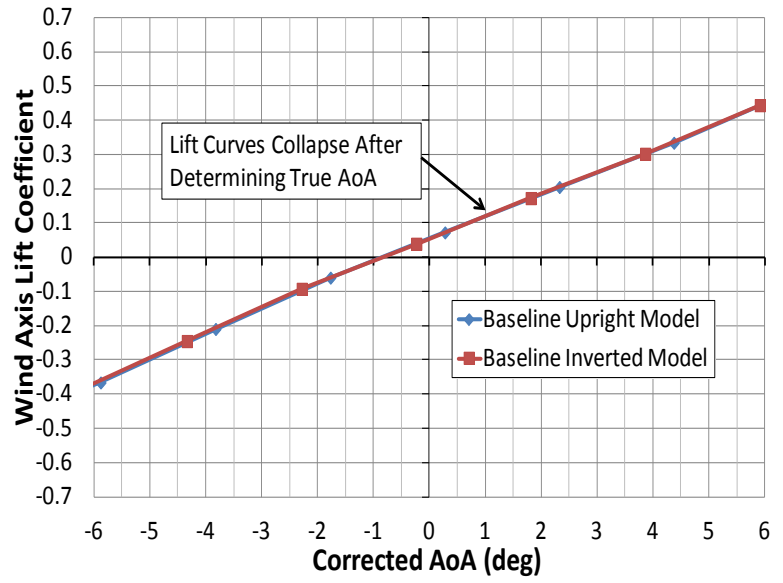

Figure 1b. Typical corrected lift curves.

Wind tunnel test engineers and customers also expect to see good upright and inverted pitching moment curve $\left(C_{m}-\alpha_{c}\right)$ agreement after applying the aforementioned upflow correction. This is not always the case, as sting interference, mount blockage, wing downwash, and longitudinal changes in tunnel flow, among other culprits, can manifest themselves in a more pronounced manner for pitching moment curves than with lift curves. The theoretical trailing vortex model proposed by Barlow, et al. ${ }^{1}$, though practical for traditional wind tunnel testing, focuses on pitching moment slope (i.e., stability) rather than the present concern of pitching moment trim (i.e., constant moment offset with AoA). The empirical pitching moment correction proposed by Heyson ${ }^{2}$ requires a tail balance (or estimation of the tail's contribution to total forces while in the wing wake). Tail balances are not always practical in wind tunnel models, and estimation of tail-in-wake forces carries uncertainties due to wind tunnel wall effects. Instead, it is desirable to use the removal and/or deflection of common 3-D aircraft model components and the inversion of said model to determine any differential flow qualities that exist between the model's wing and tail.

\section{Wind Tunnel Test Facility}

The Wichita State University / National Institute for Aviation Research (WSU NIAR) Walter H. Beech Wind Tunnel (WBWT, Fig. 2), is a closed-loop, low speed $(\mathrm{M}<0.3)$ wind tunnel, with a closed 7x10 foot test section. The WBWT's internal balance support system is in current use since its commissioning in 2007 and subsequent proof testing of existing production aircraft models ${ }^{3}$. The internal balance sting support is equipped with an image system that allows automated incidence and roll angle changes; removal of the image system permits sideslip angle change via the underfloor external balance structure. Two load range stings are available, along with

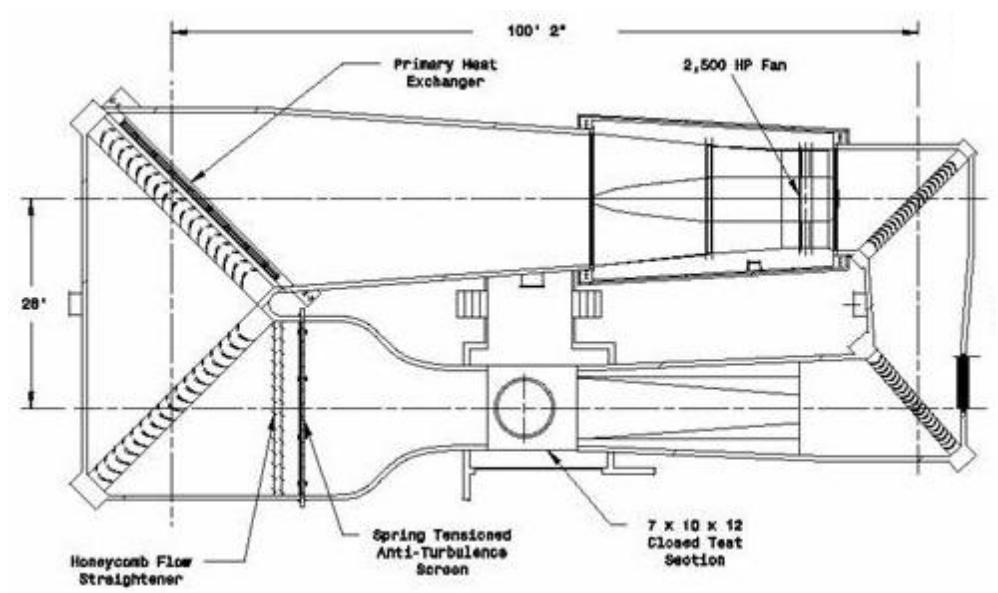

Figure 2. Walter H. Beech Wind Tunnel Plan View both force and moment design two-inch internal balances from Triumph Aerospace $^{\circledR}$ and Ate AEROTECH ${ }^{\circledR}$, respectively. The internal balance support system (Figure 3), with available stings, locates models both forward of and slightly below the center of test section. This location is set based on customer-driven design requirements for model size and AoA capabilities, as well as the cost advantage of utilizing the existing external balance structure for structural mounting, precise positioning, and sideslip motion. A correction algorithm is in place in the WBWT data reduction software for the observed variation in dynamic pressure with model longitudinal position.

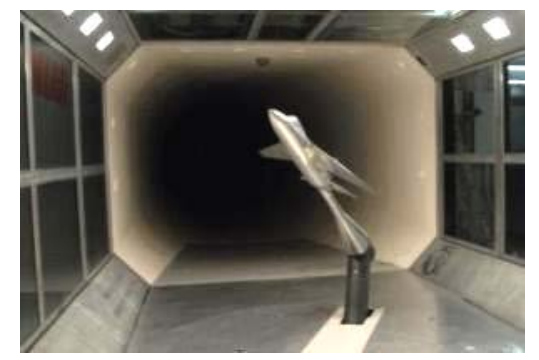

Figure 3. WBWT Sting System. 
Tests of 3-D aircraft models on the WBWT internal balance support demonstrate a significant and repeatable vertical pitching moment offset, $\Delta C_{m}$, in the moment curves of an upright and inverted model, as seen in Figure 4. This $\Delta C_{m}$ is consistent across the tunnel's set of internal balances, stays fairly constant with AoA change, and persists even after verifying good correlation in upright and inverted lift curves with standard tunnel flow and boundary corrections applied. The WBWT presented this finding at the 2012 Subsonic Aerodynamic Testing Association (SATA) international meeting ${ }^{4}$. The present low-speed wind tunnel community expressed familiarity with such data reduction peculiarities, and

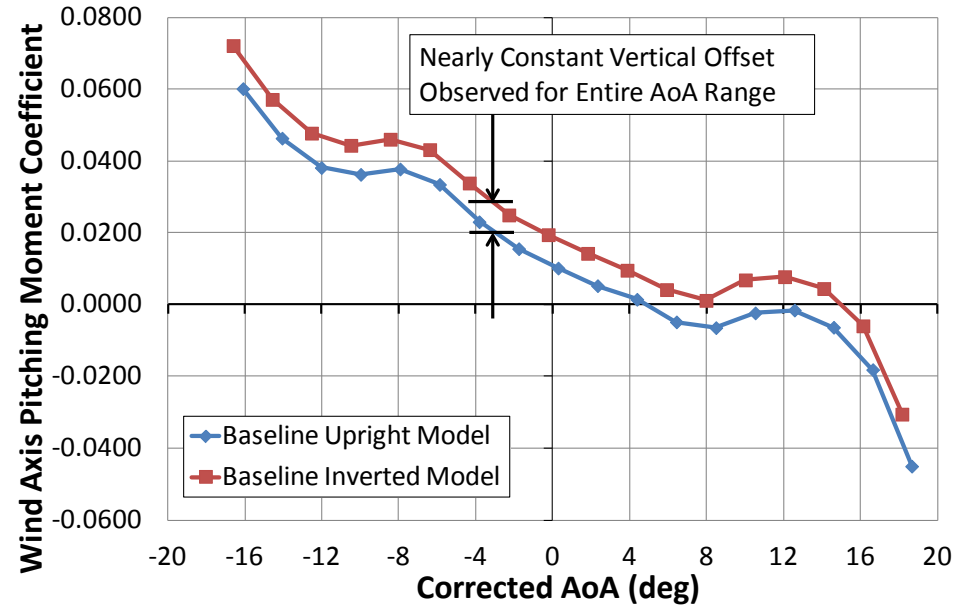

Figure 4. Typical corrected 3-D aircraft moment curves. welcomed the WBWT to investigate the pitching moment offset and present findings at a subsequent international meeting.

\section{In-House Calibration Check Models}

The WBWT possesses two internal balance-mounted 3-D aircraft models for regular calibration checks of the entire wind tunnel flow quality, data measurement, and data reduction systems. These models, known as the "Fighter-Bomber" (FB) and Super Cub, are shown in Figures 5a and 5b installed on the WBWT internal balance sting system. Both models assemble with minimal time investment and exhibit very repeatable data across multiple re-installations in the tunnel.

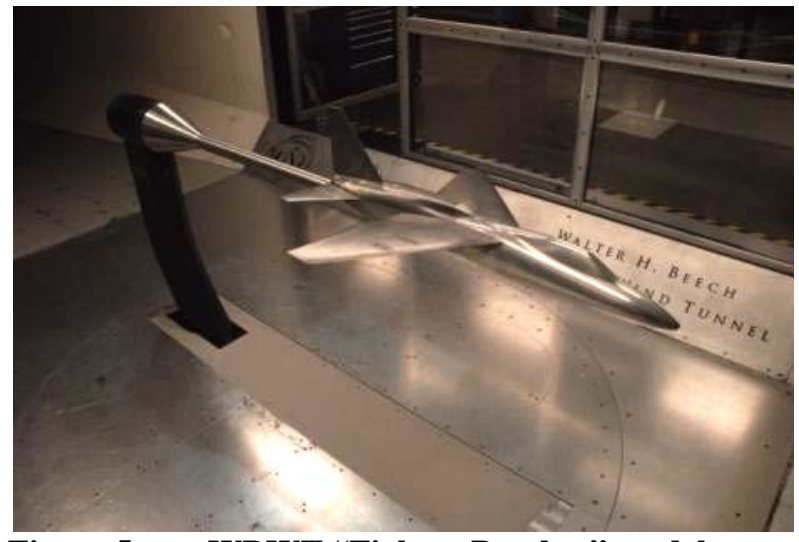

Figure 5a. WBWT “Fighter-Bomber” model.

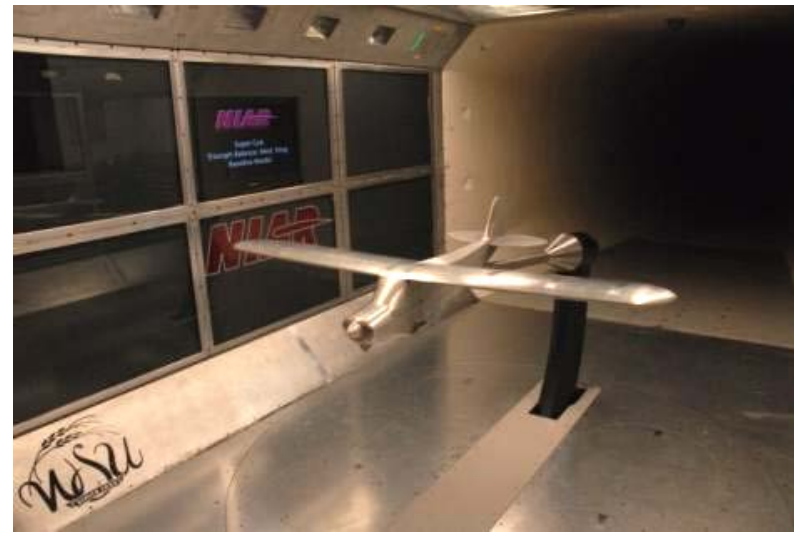

Figure 5b. WBWT Super Cub model.

The FB model features interfaces for all available WBWT two inch internal balances (both force design and moment design) and a deflectable horizontal tail (h-tail) for tail effectiveness runs. The Super Cub model only mounts to the force design 2-inch balance, and has a fixed h-tail, but features replacement "blanks" to facilitate runs with the wing and/or htail removed for component load analysis. Additionally, the Super Cub model can be mounted on the WBWT external balance via a built-in

\begin{tabular}{|r|c|c|}
\hline & "Fighter Bomber" & Super Cub \\
\hline Wing Planform Area (ft^ 2$)$ & 1.38 & 3.77 \\
\hline Wing Mean Aerodynamic Chord (ft) & 0.70 & 0.75 \\
\hline Wing Span (ft) & 2.3 & 5.0 \\
\hline Wing Aspect Ratio & 3.7 & 6.7 \\
\hline H-Tail Planform Area (ft^2) & 0.37 & 0.57 \\
\hline H-Tail Mean Aerodynamic Chord (ft) & 0.3 & 0.4 \\
\hline H-Tail Span (ft) & 1.2 & 1.4 \\
\hline H-Tail Aspect Ratio & 3.2 & 3.2 \\
\hline H-Tail Moment Arm (ft) & 1.3 & 1.8 \\
\hline Sting-Tail Volume Ratio (H-Tail / Wing) & $50 \%$ & $37 \%$ \\
\hline
\end{tabular}

Figure 6. WBWT FB and Super Cub model longitudinal geometry. 3

American Institute of Aeronautics and Astronautics 
mounting interface; due to this interface, the internal balance sting must enter the model at a significant angle relative to the wing reference plane, which requires additional data corrections for balance-to-model-body misalignment.

Figure 6 presents the pertinent wing and h-tail geometry for both calibration models. Though both models have similar wing mean aerodynamic chord and h-tail planform area values, the FB model's swept wing span and planform area are $45 \%$ and $37 \%$ compared to those of the Super Cub model, respectively. Relative to the Super Cub, the FB model features a larger h-tail volume ratio to compensate for its shorter h-tail moment arm. The vehicles are designed for very different flight regimes (i.e., fighter jet and bush plane), and their distinct aerodynamic characteristics make them robust as WBWT in-house calibration check models.

\section{Methodology and Data Reduction Checks}

Pre-test check loads, leveling a model in incidence and roll angle, and data reduction checks are all mainstays performed at the start of standard internal balance installations at the WBWT. A systemic error in start-of-test methodology can result in a number of repeatable (and undesirable) phenomenon - potentially generating the pitching moment offset observed at the WBWT for upright and inverted model runs.

To ensure methodology is not the culprit, a deliberate set of potential errors is introduced into the system, with the intention of discovering the measured response in question has either diminished, or (more likely) been magnified. As in standard statistical methods, a lack of significant observed change in the response indicates there is no direct relationship to the independent variable. Any significant changes in the response variable are further scrutinized to ensure that the set of independent variables and nuisance factors have been quantified and controlled. Furthermore, the experimental designs are randomized when applicable to prevent transient bias in the response variable.

\section{A. Pre-Test Check Loads}

Both internal balance designs available at the WBWT feature calibration blocks that enable check loadings via weight pans and check weights at precisely machined moment arms, as exemplified in Fig. 7. Basic weight checks do not indicate a pitching moment offset with balance inversion, thus a simulated "wind-on" weight check run is performed to trace any systemic errors through the entire data acquisition and reduction process. A constant differential pressure is applied pneumatically across the WBWT dynamic pressure measurement instrument to simulate wind-on conditions in the check loading data table. Subsequently, the data for upright and inverted weight check runs are acquired and reduced using the standard WBWT software and assessed for presence of a significant $\Delta C_{m}$.

Additional runs are performed where the data reduction system is "tricked" into processing and comparing check load runs that are intentionally identical, other than an "inverted model" trigger placed in one of the runs. Neither the reduction of true upright/inverted check load data nor the inverted model trigger produced $\Delta C_{m}$ values characteristic of Fig. 4.

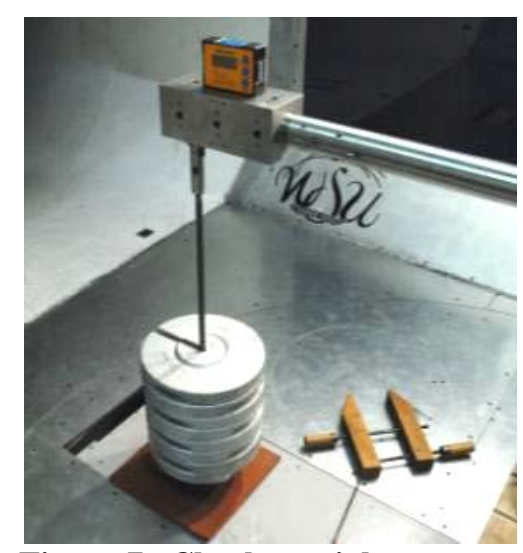

Figure 7. Check weight system. Loading shown is negative normal force and pitching moment.

\section{B. Model Leveling}

Model incidence and roll-angle leveling is typically performed via a removable, precision-ground, leveling plate that is pinned near the model's moment reference center location. An inclinometer is placed atop the leveling plate and used to precisely set motion system datum at the desired "zero" angle. Both of the WBWT in-house calibration models require wing removal to access the leveling surface, which is defined as the wing mounting plate for each model. It is preferred that the leveling plate be installed in addition to a completely assembled 3-D aircraft model, since the wing has significant mass and affects the model incidence via sting bending.

In lieu of a full-model leveling plate check, it is practical to perform the wing-off leveling, then locate and indicate a flat feature of the model upon which an auxiliary leveling can be performed. In this way, the wing-off level angle is translated to a repeatable location that is available for subsequent measurement after wing installation. The change in auxiliary reference angle after wing installation (due to wing weight) is recorded, and comparison to the wing-off auxiliary reference angle results in an absolute model incidence relative to the under-wing leveling surface. The same process is performed on an inverted model to ensure sting incidence offset (relative to the model reference plane) is not biasing the inverted model incidence - a process referred to as re-leveling. 
The WBWT Super Cub calibration model highlights the potential for error when an inverted model is not releveled - the sting enters the balance block at a 7.5-deg angle relative to the wing reference plane, as shown in Fig. 8. Thus, when the model is inverted, it obtains a 15deg true incidence angle for sting indicated 0-deg incidence. The same is true, though not nearly so visible, for models with any misalignment between the internal balance centerline and model reference level surface. It is of particular interest to assess the impact, if any, of this "balance aerodynamic misalignment" on observed $\Delta C_{m}$ for the WBWT calibration models.

Two discrete datum are chosen for setting model incidence angle, with the end goal of quantifying the effect of balance aerodynamic misalignment on the FB model's $\Delta C_{m}$. In the first approach, model 0 -incidence is set relative to the wing mounting surface, per the

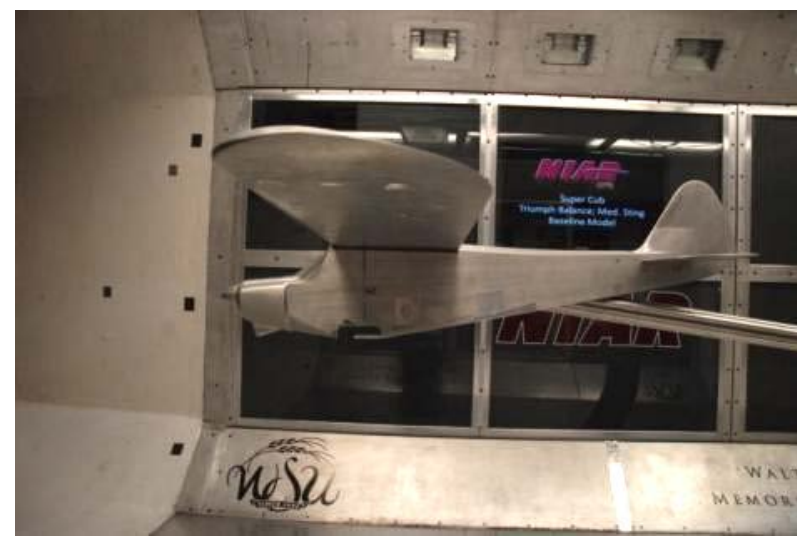

Figure 8. WBWT Super Cub model to sting interface. The 7.5-deg sting-to-model incidence stresses the importance of model re-leveling upon inversion. auxiliary leveling method described above. Upright and inverted model runs are completed, and any observed misalignment remaining will be absorbed into $\alpha_{c}$ as an empirically determined full-model upflow parameter, $\alpha_{u p}$, per classical low-speed wind tunnel corrections ${ }^{1}$. The resulting $\alpha_{\mathrm{c}}$ is applied to the upright and inverted moment curves of Fig. 9, and $\Delta C_{m}$ values are calculated. In the second approach, model 0-incidence is set such that the wing lower surface indicates an equal and opposite incidence angle when the model is inverted and no additional sting incidence datum changes are made. This differs from the typical approach in ensuring that instead of the wing, the internal balance itself is leveled, as indicated by the unchanged incidence magnitude when rolling the sting 180-degrees. Again, $\alpha_{u p}$ is determined, upright and inverted moment curves are generated, and $\Delta C_{m}$ values are calculated.

The $\Delta C_{m}-\alpha_{c}$ trends resulting from each incidence setting method are shown in Fig. 10. There is a general shift in the moment curves (equivalent for both upright and inverted cases), thus incidence setting should be monitored carefully to ensure that both the balance angle and model reference angle are known in any model roll orientation (e.g., upright and inverted roll angles of 0 and 180-degrees, respectively). Model re-leveling upon inversion is not shown to significantly impact the pitching moment differential trend with AoA. As such, the choice of model leveling method is not considered a contributing factor to the observed $\Delta C_{m}$.

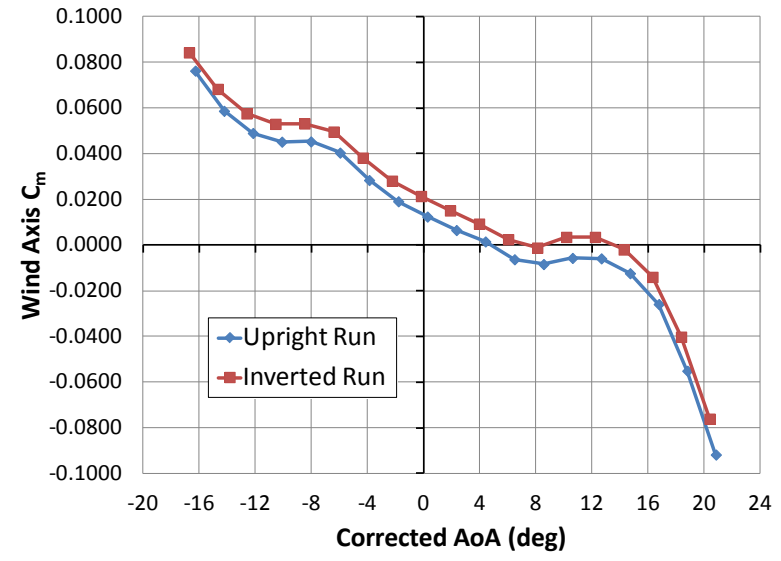

Figure 9. FB moment curves for model wing leveled while upright only. Standard WBWT flow angularity runs do not involve re-leveling model after inversion.

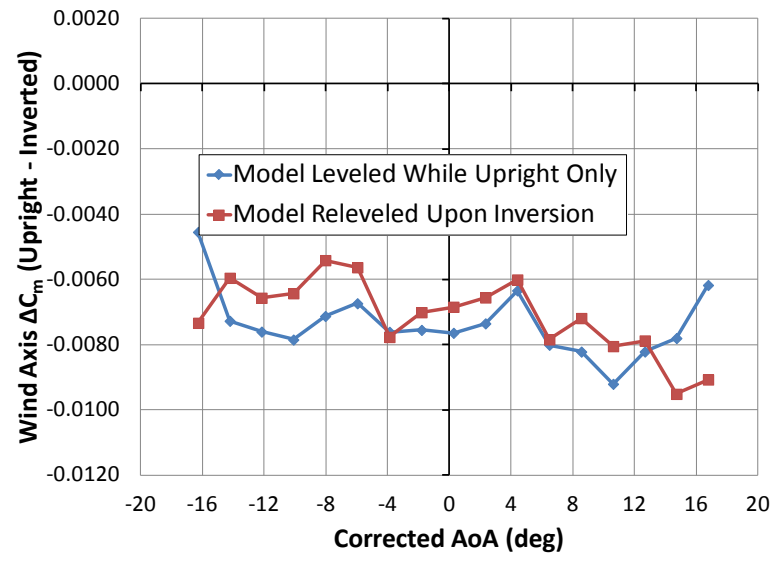

Figure 10. FB differential moment curves due to model inversion leveling method. Re-leveling model after inversion does not impact moment offset trend.

\section{Inversion of Model Only}

Inversion of a model relative to the internal balance is another method to isolate the $\Delta C_{m}$ root cause. In this procedure, a typical "baseline" run is performed in the standard fashion for a model installed upright at balance roll angle zero. Immediately thereafter, the model anti-roll pin is removed from the balance upper pin hole, the model is rolled 180-degrees, and the pin is reinstalled in the balance lower pin hole. The balance itself has not been rolled, but the model is secured for testing in the inverted position. In this way, a full mirror image is expected in the data, as 
neither the data acquisition nor data reduction systems have record of the model inversion. Any inversionspecific corrections are not applied, and the rotation of loads into the model reference plane is performed manually offline. The resulting $\Delta C_{m}$ is thusly reduced to an assessment of physical load change due to aerodynamic effects of the model and mounting structure.

The FB model is chosen for this exercise, due to the availability of WBWT comparison data for repeat installations of the FB on both available two-inch internal balances. Unlike the Super Cub model, removal of the FB model's anti-roll pin (and subsequent rotation of the model relative to the internal balance) does require removal of many model features, including the wing. However, the comparison data indicates that repeat model installations have not produced pitching moment scatter in the range of the observed upright-inverted $\Delta C_{m}$, thus component removal is not considered a significant nuisance factor in the comparison of model-only inversion runs.

The results of FB model-only inversion are presented in Fig. 11. As indicated by the $\Delta C_{m}$ trends of the standard inversion method (model and balance rolled together) and the model-only inversion runs (model rolled 180-degrees, balance unrolled), the observed $\Delta C_{m}$ appears to show no significant response to balance roll angle or data system "awareness" of model inversion. It is deduced, therefore, that the observed $\Delta C_{m}$ for the FB (and by extension, other WBWT internal balance tested models) is not an artifact of how the data reduction system handles model inverted runs, nor of physical load errors related to model inversion, but is attributable entirely to physical effects truly present in the acquired data. Sting interference and sting support structure blockage are assessed in the following section for their contribution to $\Delta C_{m}$. Longitudinal differential flow angularity effects are considered subsequently.

\section{Sting and Support Effects}

\section{A. Sting Interference}

Existing WBWT corrections account for the sting support's measured drag effect (model cavity and tail exit plane pressures are recorded). It is desirable to use the minimum sting diameter allowable for the predicted model loading requirements - this has the effect of minimizing sting interference, often to the point of making it insignificant. Tunnell ${ }^{5}$ states that, in subsonic flow, a model support sting with minimum aerodynamic interference must have a constant diameter section of at least four sting diameters behind the model base, terminating in no greater than a $16^{\circ}$ aft-body cone taper angle. Both WBWT calibration models meet the constant diameter section length criteria (11 sting diameters for the FB and 9 diameters for the Super Cub), but have a cone taper angle of $30^{\circ}$.

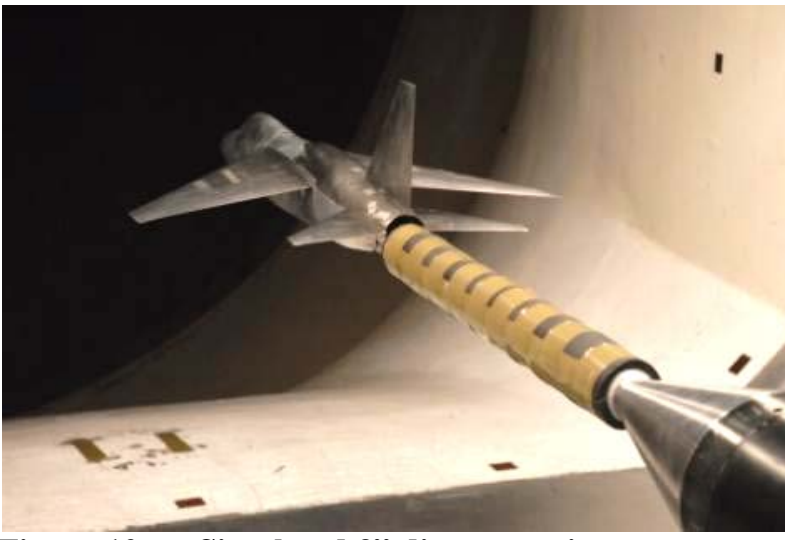

Figure 12a. Simulated 3" diameter sting.

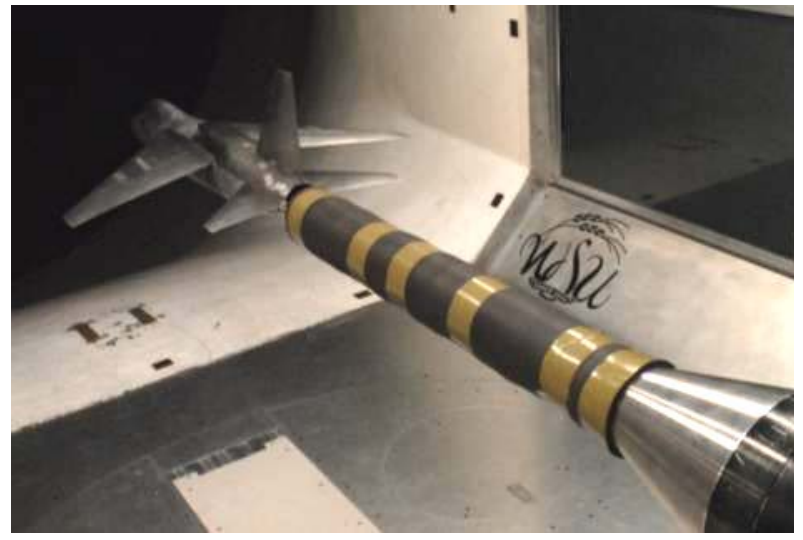

Figure 12b. Simulated 4" diameter sting.

The FB model produces fairly low loadings compared to the capability of the medium-range 2" diameter sting employed for testing at the WBWT - this is, however, the facility's smallest sting at the time of writing. In lieu of manufacturing a smaller sting and calibrating a smaller balance (a lengthy and costly process beyond the scope of 
this project), the sting interference effect on the model's aft-body and h-tail is assessed by simulating a larger sting. Pipe foam is wrapped around the sting to increase its effect on the flowfield at non-zero AoAs (and subsequently its aerodynamic interference) starting immediately aft of the model, and continuing until the sting base taper section. Diameters of 3" and 4" are simulated, as shown in Figs. 12a and 12b, respectively.

Figure 13 indicates that the $\Delta C_{m}$ trend is not consistently affected by sting diameter. Increasing sting interference does have an effect on the magnitude of pitching moment, but the effect is realized in equal magnitude for both upright and inverted models. Therefore, sting interference on the aft-body and h-tail is not likely a first-order contributor to the observed

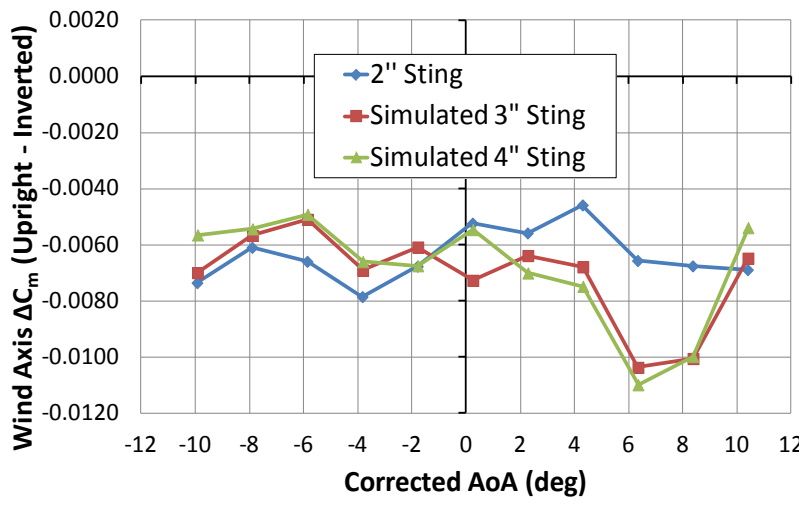
upright/inverted model pitching moment differences.

Figure 13. Sting interference effect on $\Delta C_{m}$.

\section{B. Sting Support Blockage}

Barlow, et al. ${ }^{1}$ outline a method for quantifying wind tunnel mount aerodynamic interference via a series of "image system" runs. The WBWT possesses a straight image strut as shown in Fig. 14; this strut is capable of accommodating model motion sweeps either traditionally (single axis model rotation in AoA or roll angle) or logically (simultaneous AoA and roll angle rotation to achieve sideslip angles without changing mount blockage). In theory, the image strut will equalize the mount blockage flow field effects about the model's wing plane. If the $\Delta C_{m}$ trend is due to mount blockage, then the image system diminishes the observed $\Delta C_{m}$ by influencing equivalent dynamic pressure and flow angularity above and below the sting centerline.

Figure 15 presents a comparison of FB AoA sweeps with and without image system. The image system is shown to roughly halve the pitching moment effect, but also change the sense of the differential pitching moment. Further investigation, via comparison of upright and inverted lift curves, indicates that the same effect occurs to $\alpha_{u p}$ with introduction of the image system (roughly a 50\% decrease in magnitude, and change in direction of flow angularity).

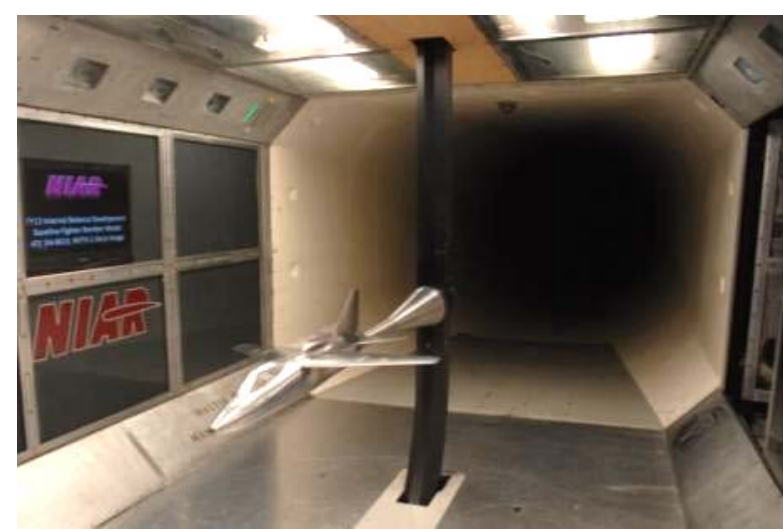

Figure 14. WBWT crescent image strut installed.

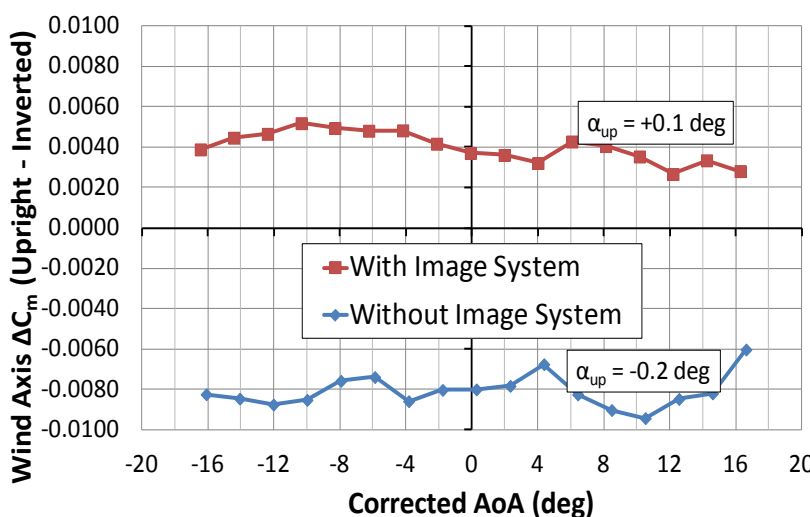

Figure 15. Image system effect on $\Delta C_{m}$.

The mount's dynamic pressure effect is not evaluated by the $\alpha_{u p}$ correction method. Instead, a NACA 16-021 ${ }^{6}$ section sting crescent fairing is chosen for its long run of favorable pressure gradient, and is fabricated of foam (Fig. 16) to temporarily minimize the mount blockage effect. Yarn tufts are applied to ensure the flow is attached for the entire fairing chord length. The foam section is rigid and does not allow traditional AoA sweeps; however, the $\Delta C_{m}$ effect is observed for the unfaired sting at 0-deg AoA, thus a single AoA upright-to-inverted comparison is sufficient to assess mount blockage effects on $\Delta C_{m}$. Figure 17 indicates that the mount blockage dynamic pressure effect is not a significant contributor to the overall value of $\Delta C_{m}$, as evidenced by comparison to the unfaired complete AoA sweep values of $\Delta C_{m}$.

Rollhead bluff body blockage is not assessed for flow-field effects, as the rollhead is axisymmetric to the sting centerline. It is assumed that the associated bluff body wake also carries symmetric effects that do not propagate into the reduced $\Delta C_{m}$ - i.e., the rollhead's flow-field effects are equivalent for both the upright and inverted model cases. 


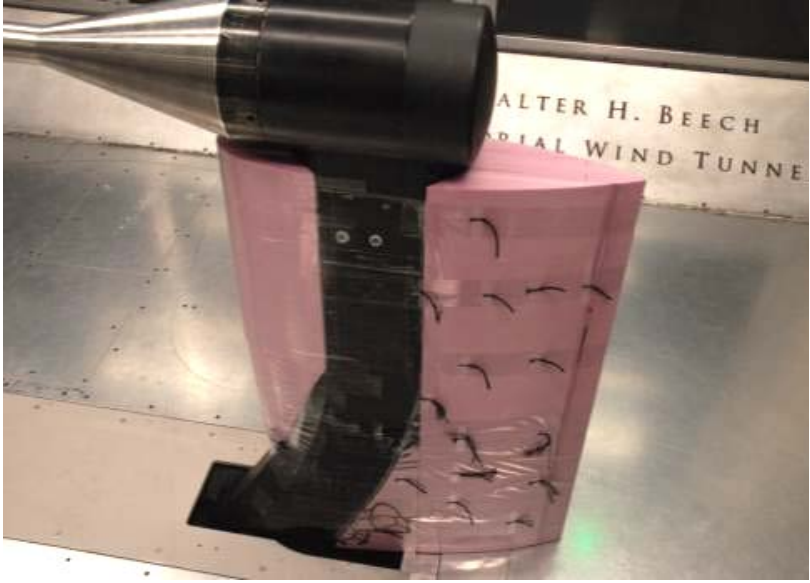

Figure 16. Faired sting crescent. Pictured wind-off.

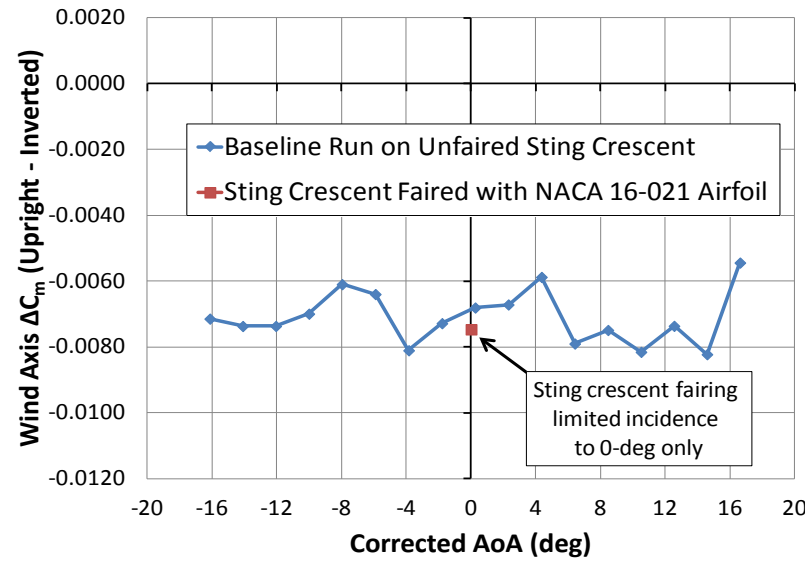

Figure 17. Sting crescent fairing effect on $\Delta C_{m}$.

\section{AoA Sweeps in Other Planes}

Performing effective AoA sweeps for a model with non-zero roll angle has the effect of minimizing or eliminating mount blockage in the model sweep plane, though the overall blockage does increase with the introduction of yaw angles. In particular for the WBWT sting support, a set of "AoA" sweeps at roll angles of 90 and 270-degrees (the "no-blockage" case, as the yawed mount blockage is not in the model wake) also eliminates any asymmetric longitudinal wall proximity effects that result from the sting being below tunnel centerline. In addition to the "no-blockage" runs, "half-blockage" AoA sweeps are performed (with model and balance roll angle set to 45 and 235-degrees in back-to-back runs) by mixing sting pitch and yaw angles to generate the desired total AoA. $\Delta C_{m}$ trends as a function of combined mount blockage, wall interference and flow field changes are formulated from these three sets of runs. The use of an automatic rollhead makes this task practical and repeatable.

Sting interference is held constant for these runs, as the entire model/sting assembly articulates as a unit and experiences the same total AoA independent of sweep plane. Physical mount blockage is (counter intuitively) the greatest for the "no-blockage" run; however, the yaw angle pivot axis is approximately midway between the model and crescent support, thus the mount blockage is not in the model's wake with increasing "AoA". This has the effect of eliminating the mount blockage from the wing and h-tail wakes. More importantly, the blockage and wall proximity are longitudinally symmetric for the "no-blockage" "upright" and "inverted" runs (i.e., 90-deg and 270deg roll angles, respectively), thus mount blockage and wall effects in the lift plane are held constant with model inversion. After eliminating sting interference, mount blockage, and wall effects by holding these factors constant, flow angularity remains as the primary known factor that varies with change in model AoA sweep plane.

The alternative AoA sweep plane $\Delta C_{m}$ results (for "full-blockage", "half-blockage" and "no-blockage", as described above) are presented in Fig. 18. The results indicate that best values of $\Delta C_{m}$ (those nearest to zero) are achieved by positioning the mount blockage out of the model's wake, and achieving symmetric tunnel geometry about the model's longitudinal axis. The $\Delta C_{m}$ effect is approximately halved by performing the "halfblockage" AoA sweep. A further implication of these results, beyond blockage and wall effects, is that flow variations (where upflow relates to AoA) exist in the "full-blockage" AoA sweep plane that are eliminated for the case of the "no-blockage" AoA sweep (where tunnel sideflow angularity relates to the model's true AoA). The flow angularity in both directions is quantifiable via 5-hole probe recordings, while the tunnel wall presence effect requires development of an

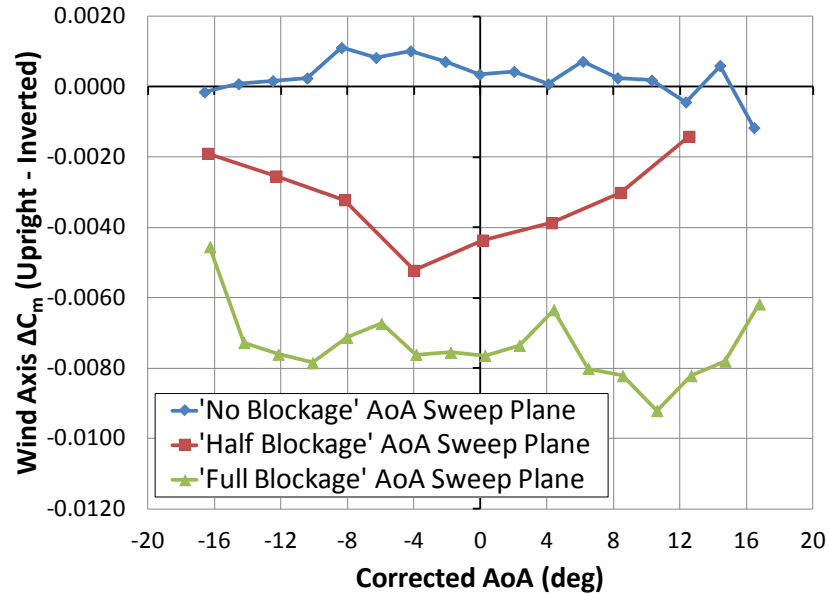

Figure 18. AoA sweep plane effect on $\Delta C_{m}$. empirical correction scheme. 


\section{Longitudinal Variation in Dynamic Pressure and Flow Angularity}

Prior WBWT investigations with Pitot-probe mounting systems have resulted in a correction algorithm for dynamic pressure variation in the longitudinal direction ${ }^{7}$. The correction is applied to the $\Delta C_{m}$ trends shown previously, and does not significantly impact the overall character of $\Delta C_{m}$. Though the longitudinal dynamic pressure variation will result in a greater airspeed over the h-tail than the wing, this change in dynamic pressure will have the same impact for upright and inverted runs, and thus is not a culprit in the $\Delta C_{m}$ trend.

A traverse-system mounted 5-hole probe is utilized to map the test section flow qualities in the presence of the sting mount (Fig. 19). The purpose of this investigation is to determine if upflow angularity has more longitudinal variation in the model's wing and h-tail region than does sideflow angularity, as is implied by the "full-blockage" and "no-blockage" plane AoA sweep results. If upflow and sideflow angularity trends are similar, the investigation's focus shifts to the only known remaining unaccounted "no-blockage" AoA sweep variable wall proximity effect due to the slightly below-centerline sting.

Figures 20 and 21 illustrate the observed upflow and sideflow angularity trends, respectively, as measured by the 5-hole probe with sting mount present in test section. Flow angularity values are presented relative to the value observed at the internal balance measurement center. The figures corroborate the assumption that flow angularity is a major contributor to the $\Delta C_{m}$ effect seen when performing the "full-blockage" and "no-blockage" AoA sweeps. Aft of the internal balance measurement center, upflow varies by approximately 0.1 to 0.3 degrees per longitudinal foot traveled, while sideflow varies by 0.0 to 0.5 degrees per longitudinal foot. Sideflow is particularly constant aft of internal balance measurement center, which represents the typical h-tail region. No major effort is taken to align the 5-hole probe with the precision required to achieve absolute angularity values, thus the flow angularity values closest to internal balance virtual center are set arbitrarily to zero, and all other results are relative to these

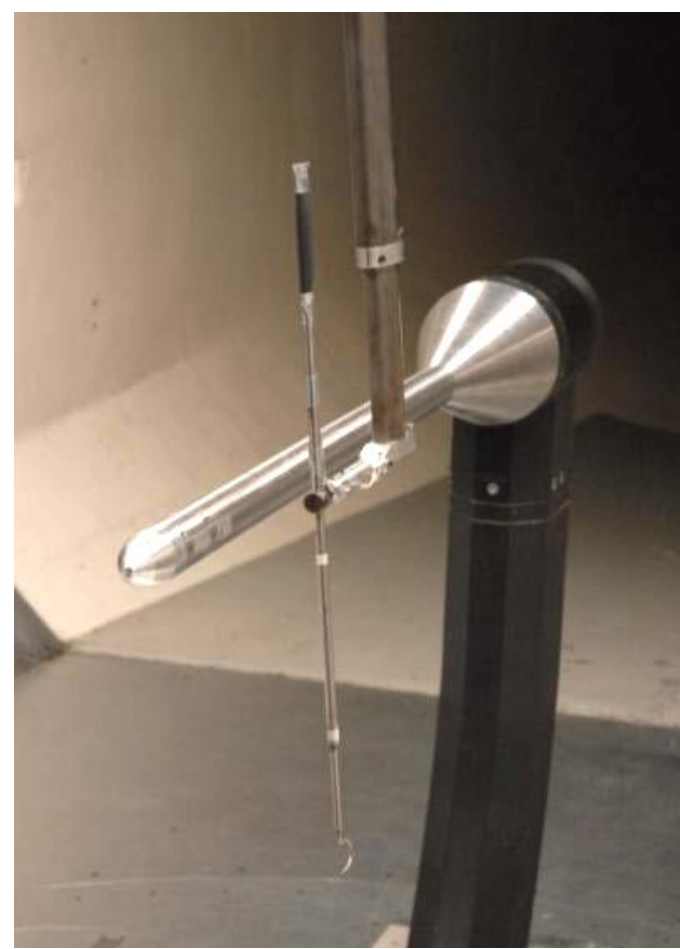

Figure 19. Traverse mounted 5-hole cobra probe setup with sting present.

"zero" values to form angularity trends. The run parameters are simplified by setting the sting incidence to zero in all cases, since the $\Delta C_{m}$ effect is observed even at zero AoA. A lateral offset distance of six inches (port direction) is chosen to keep the probe in a typical model tail span location while avoiding immediate sting interference.

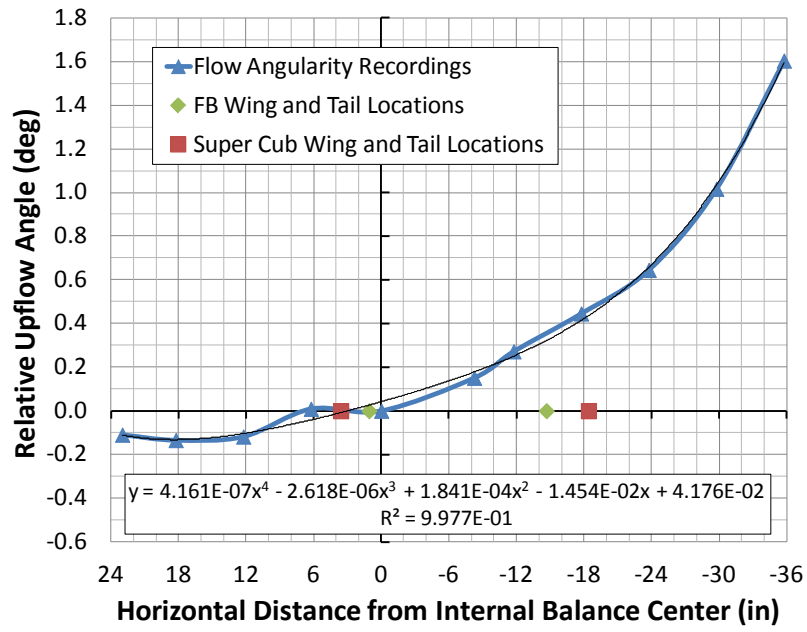

Figure 20. Upflow angle recordings at internal balance vertical centerline. Angles are relative to upflow angle observed at internal balance virtual center. All points are taken six inches port of centerline.

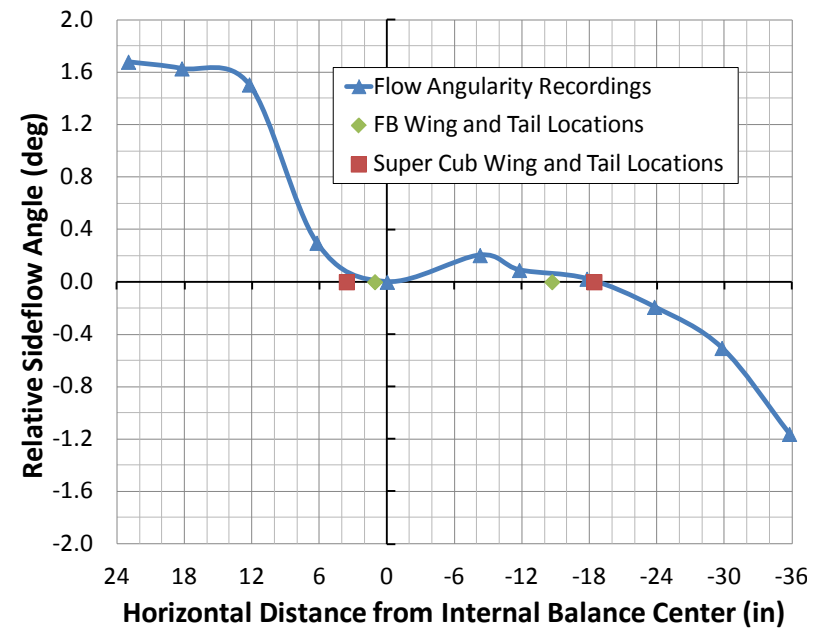

Figure 21. Sideflow angle recordings at internal balance vertical centerline. Angles are relative to sideflow angle observed at internal balance virtual center. All points are taken six inches port of centerline. 
Probe measurements are recorded at a freestream dynamic pressure of $60 \mathrm{psf}$ (3-D aircraft model data indicates that $\Delta C_{m}$ is independent of dynamic pressure beyond $40 \mathrm{psf}$, plausibly even lower) in random order at sting centerline and 12 inches above and below, and in quasi-random order at approximately 6-inch spaced longitudinal stations starting 24 inches forward and ending 36 inches aft of the internal balance measurement center. Upflow angle results at the above and below centerline positions are presented in Figs. 22a and 22b, where the angularity values shown are differences from the value observed at the internal balance virtual center longitudinal and vertical location. Trends offset from vertical centerline are critical to t-tail planforms, where flow angularity is potentially a function of tail height as well as tail longitudinal spacing relative to the wing. The below-centerline trend is necessary to handle inverted runs performed for a t-tail planform 3-D aircraft model.
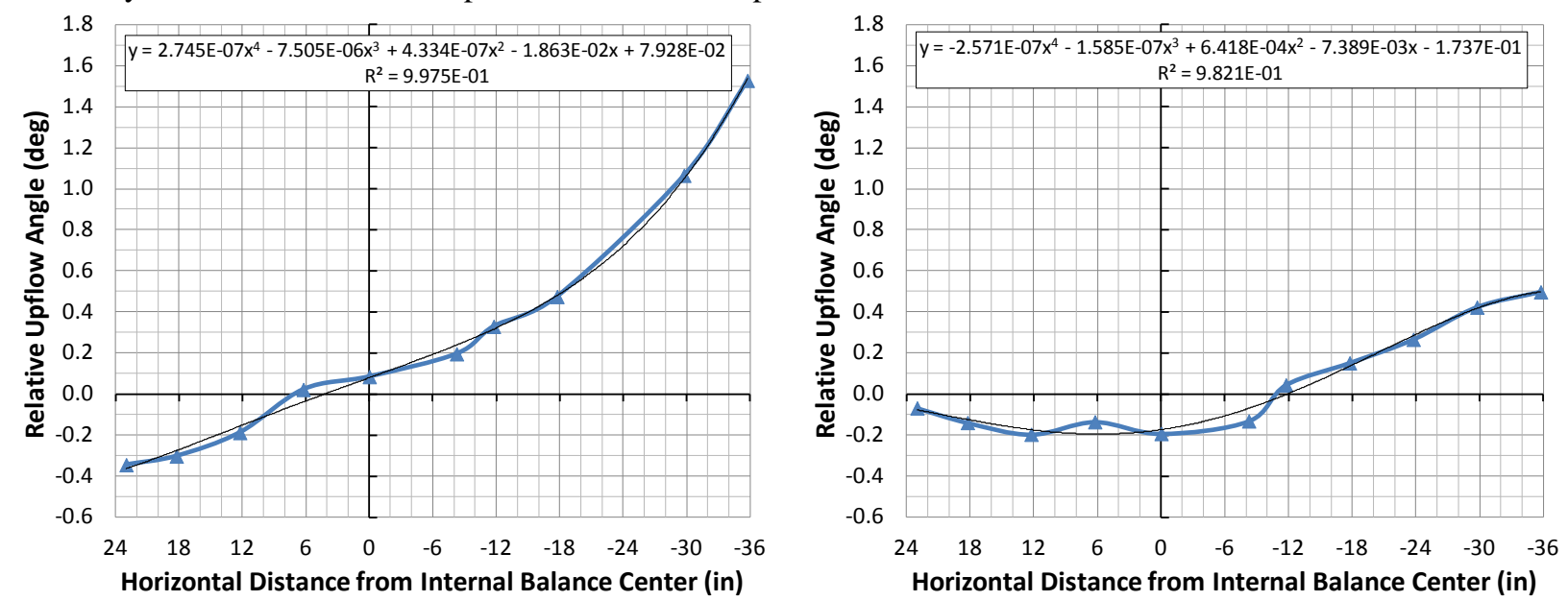

Figure 22a. Upflow angle trend 12 inches above Figure 22b. Upflow angle trend 12 inches below internal balance vertical centerline. Angles are relative internal balance vertical centerline. Angles are relative to upflow angle observed at internal balance virtual to upflow angle observed at internal balance virtual center. All points are taken six inches port of centerline. center. All points are taken six inches port of centerline.

It is apparent in Fig. 22b that the below-centerline case experiences significantly less upflow angularity variation than does either the centerline (Fig. 20) or above-centerline (Fig. 22a) case. The vertical position data are recorded in random order, thus the trends for each vertical position are obtained simultaneously (i.e., transient effects are not a factor in comparing the three vertical positions). Contrary to the present results, it is expected that the belowcenterline case exhibits the most change in flow character due to the presence of the sting blockage. Plausibly, at the position closest to the floor, the wall effect is significantly damping any upstream flow field effects of sting blockage. This theory is corroborated by the sideflow angularity observations, where the trends have the same character independent of vertical position - in this case the tunnel walls are equidistant in the sideflow direction for all three cases (i.e., changing vertical position does not move the probe any closer to the side walls, thus any blockage effects on sideflow are damped equally at all three positions).

An additional run is performed on the starboard sting side and compared to the port side results to ensure lateral probe offset is not introducing bias in the upflow observations. The starboard and port upflow results are compared in a delta table in Fig. 23. The observed differences at the lateral location are not significant compared to the longitudinal upflow angularity trends, and are within the 5-hole probe's uncertainty.

\begin{tabular}{|c|c|}
\hline $\begin{array}{c}\text { Vertical Distance } \\
\text { from Sting Centerline } \\
\text { (in) \{positive below\} }\end{array}$ & $\begin{array}{c}\Delta \boldsymbol{\alpha} \text { (deg) } \\
\text { \{Port - Starboard\} }\end{array}$ \\
\hline \hline 12 & -0.04 \\
\hline 0 & 0.02 \\
\hline-12 & -0.17 \\
\hline
\end{tabular}

Figure 23. Upflow differences for port and starboard positions at internal balance longitudinal center.

\section{Pitching Moment Correction Based on Observed Tail Flow Angularity and Tail Effectiveness}

If differential upflow at the h-tail relative to the wing is the primary cause of the $\Delta C_{m}$ trend, then this upflow change can be accounted for directly by a tail effectiveness correction. Such a correction sets the true pitching moment, $C_{m \text {,true }}$, equal to the indicated pitching moment, $C_{m \text {,ind }}$, less a term involving the differential h-tail upflow, $\Delta i_{H}$, as in Eq. 1:

$$
C_{m, \text { true }}=C_{m, \text { ind }}-\left(C_{m, i H}\right)\left(\Delta i_{H}\right)
$$


All terms of Eq. 1 are in wind axis (tunnel fixed) coordinates, and $C_{m, i H}$ is the observed change in pitching moment due to h-tail incidence angle change; determination of this term requires either two runs using an indexable h-tail or a priori knowledge of h-tail effectiveness. As the orientation of $\alpha_{u p}$ (relative to the model) changes with model inversion, the tail effectiveness term becomes additive for the inverted model case. Physically, this is to say that positive h-tail upflow for an upright model (which induces a negative pitching moment) has a decreasing effect on upright $C_{m}$, and the same positive (wind axis) upflow will have an increasing effect on inverted $C_{m}$. The tail effectiveness term in Eq. 1 carries the opposite sign to correct for pitching moment induced by upflow at the tail relative to the wing.

The FB model's indexable h-tail is utilized to determine $C_{m, i H}$ via the above method of direct $C_{m}$ observation. Comparison of the two h-tail deflection runs yields a $\mathrm{C}_{\mathrm{m}, \mathrm{H}}$ value of -0.0169 per degree of h-tail incidence. Applying the standard full-model averaged $\alpha_{u p}$ value and results from 5hole probe studies with sting present, the h-tail differential upflow, $\Delta i_{H}$, is found to be 0.22 degrees. Applying these $C_{m, i H}$ and $\Delta i_{H}$ values to the raw data, as in Eq. 1, results in the corrected data for $\Delta C_{m}$ shown in Fig. 24. These results are crossplotted against the standard reduced data (without tail effectiveness and incidence angle correction). There is a clear shift in the corrected $\Delta C_{m}$ trend (toward the desired value of zero) throughout the AoA sweep; specifically an $80-105 \%$ reduction (implying some overcorrection) for the entire run, excluding stall, for an average of $99 \%$ reduction in $\Delta C_{m}$ at non-stalled AoAs.

A run series is also performed with the sting image system installed, with results presented in Fig. 25. Interestingly, the uncorrected $\Delta C_{m}$ trend carries a near-constant value of opposite sense (though decreased in magnitude by approximately $50 \%$ ) compared to runs without the image system, as previously discussed in Section 5b. The calculated $\alpha_{\text {up }}$ follows a corresponding trend, at +0.10 degrees and -0.20 degrees for runs with and without image system, respectively. This result indicates that $\Delta C_{m}$ not only reduces when longitudinal flow angularity changes are accounted for, but that changing the flow angularity (by introducing the sting image system) induces a correctable change in $\Delta C_{m}$ consistent with the magnitude of change in $\alpha_{u p}$.

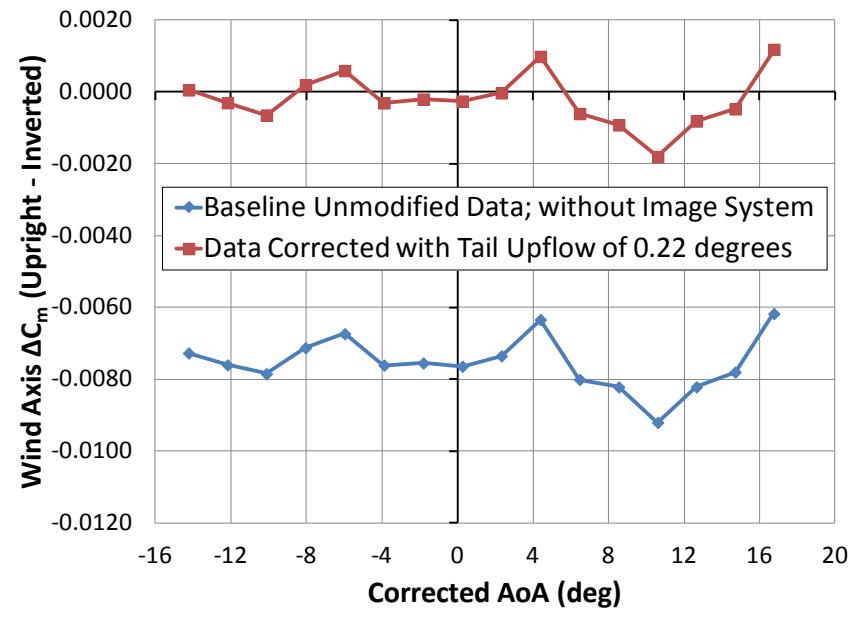

Figure 24. Effect of moment correction on baseline FB data without sting image system.

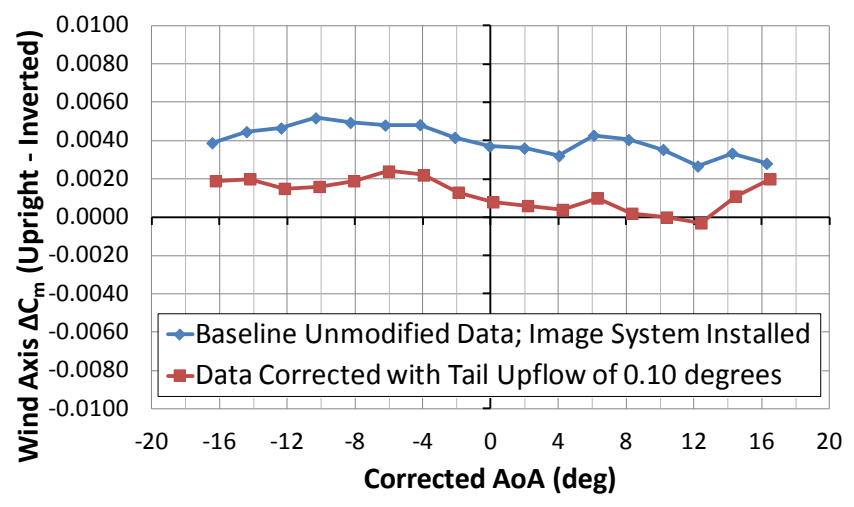

Figure 25. Effect of moment correction on baseline FB data with sting image system installed.

\section{Extension of Tail Angle and Effectiveness Correction to Other Models}

A robust correction scheme is required that, developed using the FB model, extends to the Super Cub model and other internal balance planforms. Validation is desired to demonstrate the tail effectiveness correction's functionality with models having wings of various sweep and aspect ratio, various wing-to-tail moment arms, and various airfoil sections and wing/h-tail spans. If the correction indicates good correlation for the available in-house calibration models meeting these requirements, then the WBWT can confidently extend the correction method to unknown new internal balance models encountered for funded research and development work.

\section{A. Differential Flow Angularity Prediction}

A model is developed to predict longitudinal change in flow angularity from wing to h-tail aerodynamic centers for a generic model with either conventional Blériot tail or t-tail configuration. This model replaces direct 
measurement of in situ flow angularity for funded testing, where correction development is counterproductive. Separate prediction models are presented in Eqs. 2a and 2b for conventional and t-tail configurations, with flow angularity recordings on sting vertical centerline and one-foot above centerline, respectively. Data supporting the model is presented in Figs. 20 and 22a, Section 7. The trend for flow angularity one-foot below centerline is presented in Fig. 22b, and is applicable to a t-tail model inverted run. Generally, inverted model testing is used to support development of upright model corrections only, but it may be applicable to utilize the upflow differences above and below sting centerline in ensuring pitching moment collapse for t-tail models.

$$
\begin{gathered}
\Delta i_{H, \text { conventional }}=4.161\left(10^{-7}\right) x^{4}-2.618\left(10^{-6}\right) x^{3}+1.841\left(10^{-4}\right) x^{2}-1.454\left(10^{-2}\right) x+4.176\left(10^{-2}\right) \\
\Delta i_{H, t-\text { tail }}=2.745\left(10^{-7}\right) x^{4}-7.505\left(10^{-6}\right) x^{3}+4.334\left(10^{-7}\right) x^{2}-1.863\left(10^{-2}\right) x+7.928\left(10^{-2}\right)
\end{gathered}
$$

where $\Delta i_{H}$ (degrees; upward is positive) is the difference in upflow angle of the lifting surface (wing or tail) aerodynamic center compared to that of the balance measurement center, and $\mathrm{x}$ is the longitudinal distance (inches; forward is positive) from balance measurement center to lifting surface aerodynamic center. These equations serve as estimators for differential upflow when it is not practical to directly measure the observed tail flow angle via wing-off runs, a method for which is demonstrated in Section 9c. Wing upflow is typically similar to observed $\alpha_{u p}$.

\section{B. Validation Model}

The Super Cub model is utilized to validate the tail effectiveness correction, due to its fundamental planform and size differences as compared to the FB model. The Super Cub wing airfoil is similar to a Clark-Y section, while the h-tail cross-section is a flat plate. The wing is unswept and has a much higher aspect ratio than that of the FB wing (6.7 and 3.7, respectively). Finally, the h-tail moment arm is approximately $70 \%$ greater than that of the FB (22 inches from Super Cub wing quarter chord to h-tail quarter chord, versus 15.25 inches for the FB). The differences presented above are aerodynamically significant, particularly in the presence of wall boundaries and longitudinal flow variations. If accounting for longitudinal flow variation provides a sufficient correction (at least an $80 \%$ reduction) to $\Delta C_{m}$ across the AoA range (excluding the onset of stall behavior), then unaccounted asymmetric wall boundary effects can be neglected from the present correction method development.

\section{Super Cub Component Contributions to $\Delta C_{m}$}

Typical wind tunnel models do not feature "blanks" that permit complete removal of lifting surfaces and replacement with faired shapes. The Super Cub model, however, accepts a wing blank and an h-tail blank to permit component level testing. Lifting component contributions to $\Delta C_{m}$ are assessed individually with a series of upright and inverted run configurations - wing only, h-tail only, and all lifting surfaces present. This procedure is fairly consistent with models that do permit component build-up testing, and results of introducing the h-tail are utilized to calculate $C_{m, i H}$ for tail effectiveness correction. It is preferred to test the h-tail at a few incidence angles to calculate $C_{m, i H}$, but the Super Cub model has fixed h-tail incidence. In lieu of h-tail deflection, the tail-off and tail-on runs are compared for changes in pitching moment. The htail's pitching moment effect at zero incidence is subtracted from all other AoA results to determine tail effectiveness (in the presence of the wing).

A non-standard feature of lifting surface blanks is the ability to determine the effective flow angle at each lifting surface individually. In the case of the Super Cub, the standard $C_{L}-\alpha_{c}$ plot collapse is forced by determining $\alpha_{c}$ for each configuration separately. A coupling is observed between the lift curve trends and moment curve trends for singlecomponent testing, in that if $\alpha_{c}$ is determined for the present configuration directly (not the entire vehicle, as is the norm) then the $\Delta C_{m}$ effect is also practically eliminated, as shown in Fig. 26.

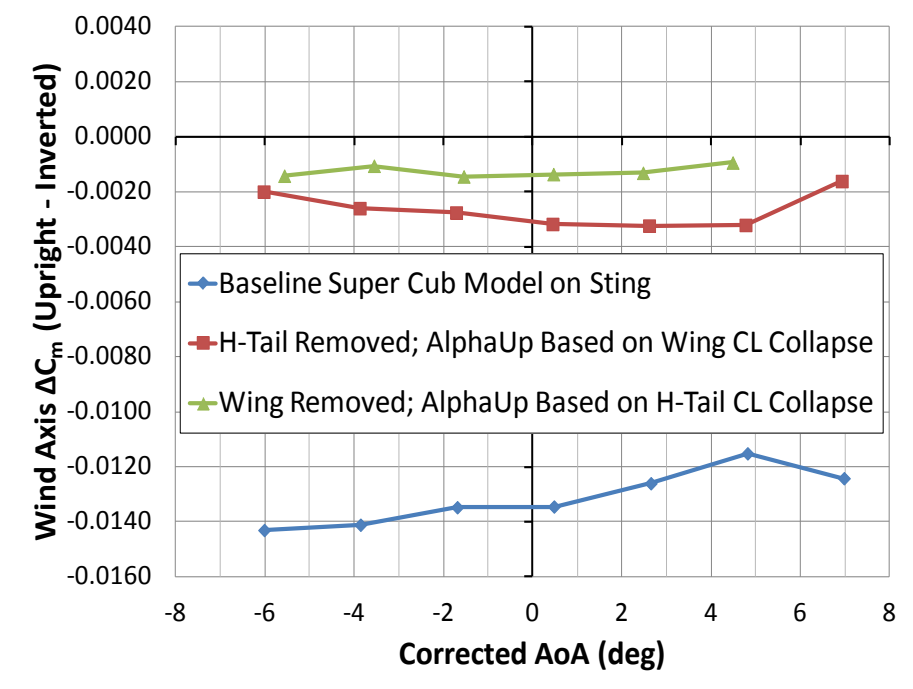

Figure 26. Effect of Super Cub components on observed model inversion pitching moment differences. 


\section{Effect of Pitching Moment Correction for the Super Cub Calibration Model}

Uncorrected and tail effectiveness corrected Super Cub $\Delta C_{m}$ trends are presented in Fig. 27, which indicates an $80-95 \%$ reduction in $\Delta C_{m}$ across the tested AoA range - consistent with the FB correction. However, there is an overcorrection, indicating that plausibly the tail effectiveness or differential h-tail upflow are overestimated. Values for tail effectiveness, $\mathrm{C}_{m, i H}$, and differential h-tail upflow, $\Delta i_{H}$, are -0.0197 per degree and 0.375 degrees, respectively. The method of tail-on, tail-off runs is employed to determine $C_{m, i H}$. The standard $C_{L}-\alpha_{c}$ plot collapse method is employed to determine $i_{H}$ for the wingoff /tail-on configuration. As with the FB corrected results, the remaining $\Delta C_{m}(<0.0030)$ is generally acceptable for corrected data, and is plausibly due to indirect interactions (e.g., wall effects, wing downwash, etc.) and experimental error. A

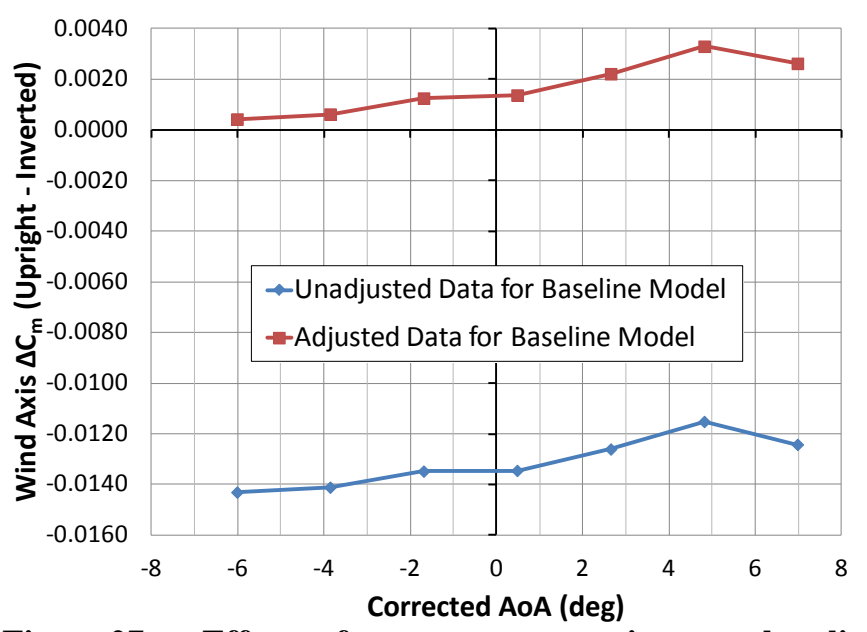

Figure 27. Effect of moment correction on baseline Super Cub data without sting image system.

standard corrections check plot set is shown in Figs. 28a and 28b, which demonstrate the standard corrections data in comparison to the present tail effectiveness correction for resulting Super Cub lift and moment curves. These plots are more typical to what the WBWT customers utilize for corrections checks, and indicate that with the present correction, good correlation is seen for pitching moment data recorded with a model upright and inverted.

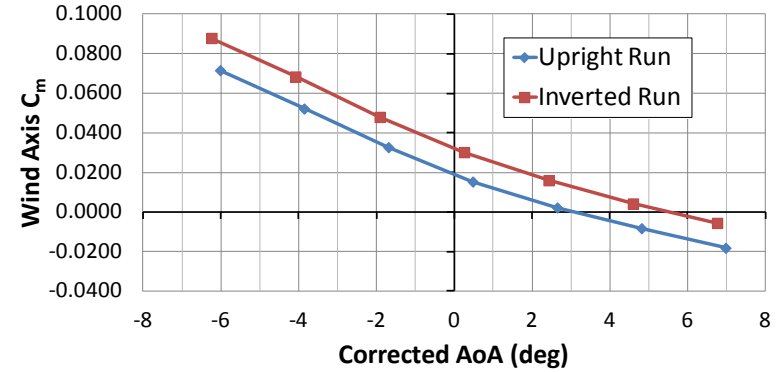

Figure 28a. Super Cub moment curves using standard WBWT Corrections.

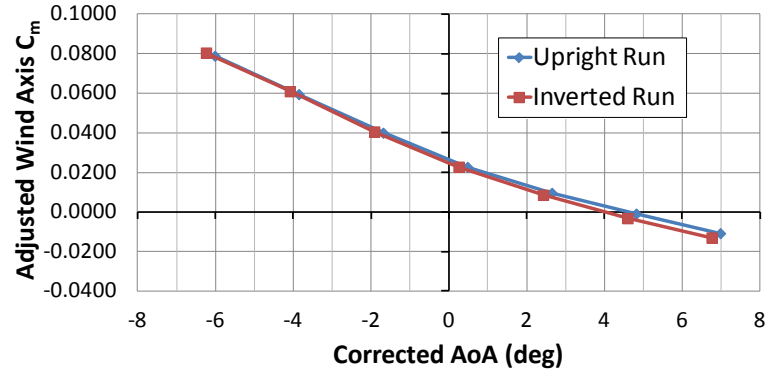

Figure 28b. Super Cub moment curves including the present tail effectiveness moment correction.

\section{E. Extension to an Atypical 3-D Aircraft Planform}

The WBWT hosts a variety of tests, including atypical 3-D aircraft models. Recent data for a lifting body/canard configuration indicates a pitching moment offset. This particular model features an indexable canard, which permits determination of $\mathrm{C}_{m, i H}$ to complement the differential tail (canard) upflow prediction model of Eq. 2a. The observed value of $\mathrm{C}_{m, i H}$ and the predicted value of $\Delta i_{H}$ complete the tail effectiveness correction of Eq. 1. Figures 29a and 29b below demonstrate the robustness of the correction method for planforms very different from the WBWT calibration models.

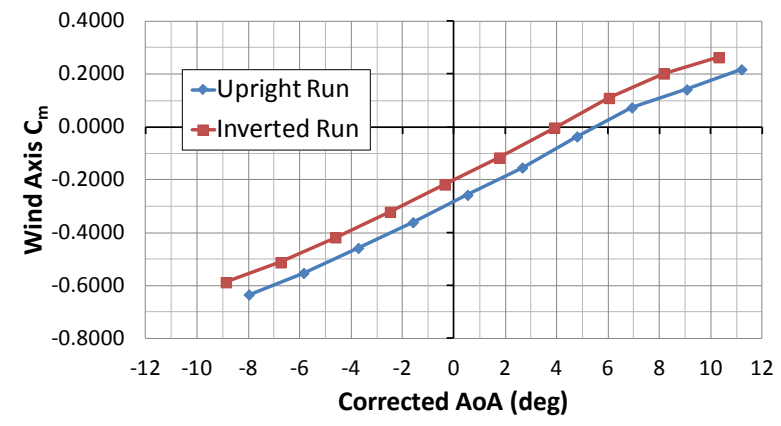

Figure 29a. WBWT proprietary model moment curves generated with standard WBWT corrections.

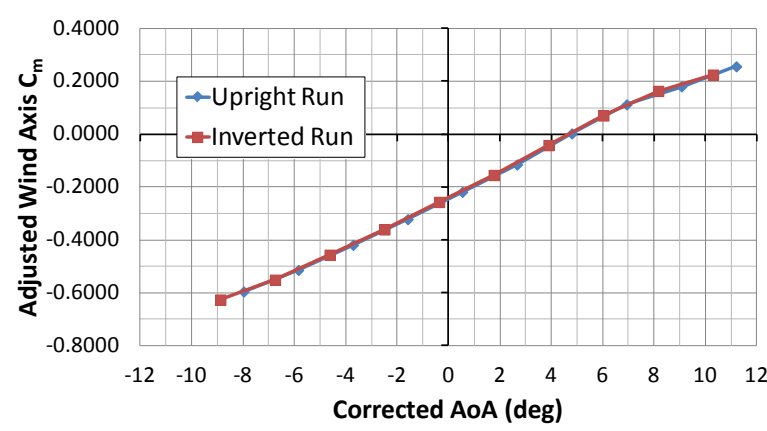

Figure 29b. WBWT proprietary model moment curves resulting from tail effectiveness correction. 


\section{Limitations and Assumptions}

The proposed tail effectiveness pitching moment correction is valid within a specific set of limitations, as follow:

1. Dynamic pressures are greater than or equal to $40 \mathrm{psf}$. Lower dynamic pressures (particularly below 20 psf) are observed to exhibit significant differences in WBWT test section flow trends such as dynamic pressure variation, position-averaged flow angularity, and turbulence intensity.

2. Sting crescent image system is not installed. Although the WBWT image system affects observed pitching moment and full-model averaged flow angularity for 3-D aircraft, the image system is unused in external client testing at the time of writing. The present correction is not validated for image system installed, and needs extension to this case should clients begin to employ the image system.

3. The 3-D aircraft is not of t-tail configuration. An upflow model is proposed for t-tail configuration 3-D aircraft, but is not yet validated. It is critical that the present correction be approached with care until such time that its effect on observed t-tail configuration pitching moment is well-understood and considered validated as a final data correction.

Additionally, a series of assumptions are applicable to the present work, as follow:

1. Tail effectiveness, $C_{m, i H}$, is reasonably constant across the tested angle of attack and sideslip angle ranges. A single tail effectiveness value is applied for all conditions to limit the amount of time required for during-test correction quantification.

2. Tail effectiveness, $C_{m, i H}$, is reasonably constant for all wing flap setting cases.

3. Longitudinal variation in WBWT upflow is consistent for sting support at zero degree sideslip as well as at non-zero sideslip angles.

4. The longitudinal location of observed model-averaged upflow is known (on a conventional planform this is typically near the wing aerodynamic center). The distance from this point to the tail aerodynamic center is the driving factor in differential upflow calculation.

5. Wind tunnel wall boundary effects on observed wing downwash and effective camber do not significantly affect the tail effectiveness or longitudinal variation in upflow.

6. The effect of boundary proximity differences on observed pitching moment for upright and inverted model testing (due to the WBWT sting being slightly below tunnel centerline) is small compared to the effect of differential flow angularity. This may not hold true for significantly large models (wing planform area exceeding approximately $10-15 \%$ of the test section cross sectional area) as the boundary effects and ground effect are enhanced.

\section{Conclusion and Recommendations for Further Work}

A series of experiments conducted at the WBWT indicate that the upright-to-inverted pitching moment offset observed in internal balance testing is due to upflow variations along the model's longitudinal axis. This is deduced after exclusion of many plausible causes, including: balance data accuracy, model incidence setting and consistency upon inversion, systemic data reduction errors involving the handling of inverted runs, unaccounted sting interference effects, and sting blockage effects. Sweeping the model through angles simulating AoA in alternative tunnel planes (e.g., performing a sideslip sweep with model rolled 90-degrees to simulate an AoA sweep) reveals that the pitching moment differential is an artifact of the sweep plane, which narrows the probable causes to wall effects (the test section is rectangular, so wall effects vary with model roll angle) and flow field phenomenon.

The flow field is quantifiable via probe measurements for dynamic pressure and flow angularity - such a quantification exercise reveals a variation in flow angularity along the model's longitudinal axis. After accounting for this variation and applying it to tail effectiveness specific to the model under testing, good upright-to-inverted pitching moment correlation is seen for a variety of planforms at the WBWT.

Further work is recommended in the remainder of this section, including: extension of the present correction to the lift and drag coefficients, as well as to V-tail planforms, empty WBWT test section flow angularity mapping, and finally, to ensure that WBWT external balance testing does not exhibit the same pitching moment offset trends.

Although a typical h-tail affects full vehicle $C_{m}$ much more than full vehicle $C_{L}$, there may exist an h-tail lift differential present with model inversion (again, due to unaccounted h-tail differential upflow angle upon model inversion). This $C_{L}$ effect would most likely be absorbed (falsely) into $\alpha_{c}$ while applying the $C_{L}-\alpha_{c}$ collapse method to determine upflow - the (possibly negligible) effect of this is to exaggerate the correction to AoA, which would propagate into all full vehicle AoA trends. Exaggerated correction could be avoided if h-tail lift effectiveness is determined in addition to h-tail moment effectiveness. Knowing h-tail lift effectiveness and h-tail upflow angle would yield a (presumably very minor) correction to full vehicle lift before applying the $C_{L}-\alpha_{c}$ collapse method to determine full vehicle upflow. 
In addition to the observed pitching moment offset trends, there have been internal balance 3-D aircraft tests at the WBWT that exhibit a lack of correlation for upright and inverted drag polars. This could be a procedural error involving failure to re-level a model when it is inverted - as mentioned in Section 4b. It could also be that the h-tail upflow differential for upright and inverted runs is further manifesting itself as trim drag in the full vehicle data.

If investigation of the lateral/directional load trends indicates a model inversion trend, it may be straightforward to develop a correction (similar to the present topic) for vertical tail effectiveness and model differential sideslip angle. However, the vast majority of 3-D aircraft concentrate their vertical tail(s) (conventional or V-tail) in a single region. For these models, a simple correction to "true" sideslip angle should suffice if performed in the manner of the $C_{L^{-}} \alpha_{c}$ collapse method, using side-force and sideslip angle as the plotting variables. An exception would be the case of a flying wing with wing-tip vertical stabilizers, where a lateral differential in sideslip angle would affect full vehicle yawing moment. In this specific case, the procedure of the present pitching moment correction method could be applied to the yawing moment via differential sideflow angle at the vertical stabilizers.

The present correction addresses only longitudinal load corrections. The case of a V-tail design would violate this limiting assumption, as the tail upflow differential would impact both longitudinal and lateral-directional loads via the V-tail's load angle components. However, it would presumably be very straightforward to determine V-tail effectiveness in the same manner as an h-tail. This effectiveness would then be separated into longitudinal and lateral/directional effects via trigonometry. Additionally, V-tails are close-coupled and thus will not encounter a variation in flow angularity relative to each other; as a result, the WBWT's standard "true" sideslip angle method should account for differential yawing moment via upright and inverted runs. Laterally, V-tail lift vectors directly oppose each other, thus a correction to rolling moment should not be necessary, regardless of flow angularity.

Finally, it is recommended that the WBWT performs a flow angularity map of the empty test section, and separately verifies with an external balance 3-D aircraft test that the $\Delta C_{m}$ trends are acceptable and do not require extension of the internal balance correction to external balance testing. At present, flow angularity evidence suggests that the internal balance mounting system (due to its significant asymmetric blockage, forward longitudinal location in test section, and vertical offset from tunnel centerline) exhibits a significant effect on tunnel upflow significantly upstream of the crescent mount. Vertical centerline located mounts faired with windshields, as is common for external balance mounting, may have a much smaller effect. Verification that there is no significant $\Delta C_{m}$ trend requires an external balance mounted, invertible model that accommodates an image system. It may be more straightforward to perform 5-hole probe testing to determine if significant longitudinal flow angularity variation trends exist in the vicinity of a representative external balance mounting system.

\section{Acknowledgments}

The author would like to thank the WBWT staff for permitting and supporting the series of testing necessary to develop the present correction. Additional thanks goes to Dr. Scott Miller and Dr. Eddie Irani for advising the tail effectiveness correction method development and implementation, as well as to the Fall 2012 AE 512 group who shared their WBWT testing time to provide FB model runs on the WBWT external balance. Final thanks to James Tennant for post-processing the sting crescent foam fairing CAD model into CNC foam cutter machine language and for assisting in installation of the fairing at the test facility.

\section{References}

${ }^{1}$ Barlow, J. B., Rae, W. H., and Pope, A., Low-Speed Wind Tunnel Testing, $3^{\text {rd }}$ ed., Wiley-Interscience, New York, 1999, pp. 271-279, 376-410.

${ }^{2}$ Heyson, H. H., "Equations for the Application of Wind-Tunnel Wall Corrections to Pitching Moments Caused by the Tail of an Aircraft Model,” NASA TN-D-3738, 1966.

${ }^{3}$ Irani, E., and Laffen, J., "Lessons Learned during Recent Upgrade: The Rejuvenation of the Wichita State University's Walter H. Beech 7x10 foot Low Speed Wind Tunnel at the National Institute for Aviation Research," $48^{\text {th }}$ AIAA Aerospace Sciences Meeting, AIAA-2010-1487, AIAA, Orlando, FL, 2010.

${ }^{4}$ Laffen, J. W., “On Balance Aerodynamic Alignment and Moment Corrections,” SATA 2012 Meeting, SATA, Tokyo, Japan, 2012.

${ }^{5}$ Tunnell, P. J., “An Investigation of Sting-Support Interference on Base Pressure and Forebody Chord Force at Mach Numbers from 0.60 to 1.30,” NACA RM-A54K16a, 1955.

${ }^{6}$ Abbott, I. H., and von Doenhoff, A. E., Theory of Wing Sections: Including a Summary of Airfoil Data, Dover Books on Aeronautical Engineering, Dover Publications, New York, 1959, pp. 333.

${ }^{7}$ Deneault, N., Kuenn, A., Kelly, K., Wong, J., and Irani, E., "Integration of Test Capabilities at the WSU/NIAR 7x10 Foot Low Speed Wind Tunnel Using CFD Methods,” 50 ${ }^{\text {th }}$ AIAA Aerospace Sciences Meeting, AIAA-2012-927, AIAA, Nashville, TN, 2012. 